\title{
REFORMA AGRARIA: UN MIEDO DE ANTIGUO RÉGIMEN EN UNA SOCIEDAD NEOLIBERAL
}

Alejandra Araya Espinoza 



\section{REFORMA AGRARIA: UN MIEDO DE ANTIGUO RÉGIMEN EN UNA SOCIEDAD NEOLIBERAL ${ }^{1}$}

Los 50 años de la Reforma Agraria hoy se conmemoran en torno a la ley 16.640 promulgada por el Presidente Eduardo Frei Montalva, pero fue en el gobierno del Presidente Salvador Allende que su aplicación tuvo mayores consecuencias políticas, convirtiéndose en uno de los temas más sensibles de la reacción conservadora a su gobierno. Dicha reacción, por cierto, se sumaba a la aversión por las clases populares y en particular la inversión de mundo que representaba el campesino organizado y con derechos laborales. Los documentos seleccionados para este dossier inician en los llamados “albores de la República”, puesto que la cuestión de la concentración de la propiedad de la tierra, la defensa de la propiedad privada y las expropiaciones por causa de utilidad pública son aspectos centrales para configurar el territorio del nuevo Estado y su tesoro, es decir, el Fisco. Las reformas agrarias, lo dicen los más variados autores, fueron eso, reformas y no procesos revolucionarios de cambio. El golpe de Estado del 11 de septiembre de 1973 y el inicio de la dictadura cívicomilitar demoniza la vertiente de cambio social y pérdida de los bienes por vía de la "rapiña" de los "upelientos", pero profundizó la expropiación de los bienes con fines de utilidad pública para la instalación de la libre explotación privada de los bienes públicos. Los estudios publicados en este volumen aportan con mucho al análisis de este proceso resguardado por la Constitución vigente.

La política en nuestro país tiene una afincada y férrea relación con la defensa de los poderes tradicionales, cuyos símbolos más inamovibles se encuentran en la tierra y las formas de la vida señorial. La inversión en tierras como signo de estatus es una de las estrategias más vigentes de nuestra movilidad social. El primer conjunto de textos seleccionados explora esas seminales discusiones del Antiguo Régimen a la República, en que las cartas sobre la mesa se expusieron con todas sus letras: el

1. Esta introducción al dossier histórico fue escrita por mí, pero la autoría en la formulación de las perspectivas de lectura y la selección de los textos es un trabajo colectivo junto al grupo de historiadores que conforma el Área de Investigación y Patrimonio del Archivo Central Andrés Bello: Ariadna Biotti, Tomás Cornejo, Natalie Calderón y Tamara Araya. Durante todo 2017 se trabajó en conformar un corpus de textos fundamentales para la historia de la Universidad en Chile y de la Universidad en Chile, repertorio a los cuales pertenecen los que se reproducen en esta ocasión y que también se encuentran en línea y accesibles desde el Catálogo Bello de nuestra Universidad (http://catalogo.uchile.cl). Agradecemos al SISIB, y en especial a su Directora Gabriela Ortúzar, el apoyo a esta empresa. 
poder privado, o el poder público. Los mayorazgos eran una tradición de traspaso de propiedad de un pedazo de tierra por vía de herencia con el objeto de controlar la división de la propiedad, razón por lo cual pasaba al hijo "mayor". Algunas veces el "vínculo" estaba asociado a un título nobiliario. Mundialmente la institución empieza a ser criticada de la mano de las teorías capitalistas liberales de fines del siglo XVIII que abogan por la libre circulación de los bienes y personas. La relación entre posesión de tierras y "aristocracia" fue duramente criticada por la Revolución Francesa que asoció dicho vínculo con un régimen social injusto y desigual, abusivo e intolerable en una sociedad de iguales en derechos. Las independencias de los países latinoamericanos formaron parte de estas mismas discusiones, pero los "revolucionarios" encontraron en este punto una férrea oposición. Bernardo O’Higgins propuso la abolición de los mayorazgos en 1818 y no tuvo éxito; la idea se repuso en 1825 y 1826.

A este debate corresponden los dos primeros textos de este dossier elegidos por la claridad de sus conceptos y de los elementos que están en juego en la negociación política que por cierto fracasa definitivamente cuando en la Constitución de 1828 se consagra la abolición de los mayorazgos y entonces estalla la guerra nuevamente. Uno se titula así, "Mayorazgos", y es un anónimo fechado en 1826 que propone "aplicar el derecho de propiedad a la subsistencia de los vínculos", ya que estos no son más que una "herencia testamentaria en usufructo". Respecto de la crítica social a esta institución, basada en privilegios y sostenedora de una aristocracia fuera de lugar dentro de una República, el texto replica que los 17 mayorazgos existentes no sustentaban una aristocracia, pues no era la generación de riqueza su principal fin y que debieran sus dueños ser considerados capitalistas si se era fiel al principio de que la acumulación, en las nuevas ideas, era un bien:

"Si un capitalista es á los ojos de los economistas la alhaja mas preciosa de un Estado. -Si segun ellos valen mas á la producción, como á la riqueza pública y particular mil pesos, por ejemplo, puestos en una mano hábil, que la misma cantidad repartida en otros tantos individuos de igual habilidad, por razones que no podemos estendernos. -Si en fuerza de ese papel que hacen en la produccion los capitales, miran esos sabios las acumulaciones como un bien, y la disipacion como un mal, hasta el estremo que uno de los mas célebres compare al pródigo con el administrador infiel de una obra piadosa, es evidente que estas instituciones que en Chile solo tienden á mantener una moderada acumulacion, llenan todos los objetos que en esta parte puede proponerse a un legislador. Que se dijese lo contrario en un tiempo en que no se comprendia como el capital que pertenece á un individuo puede concurrir á enriquecer á otros -en que se creía 
que no podia ganar uno sino lo que otro perdiese \& \& ya se podria sufrir, pero no en el diá en que se han ractificado las ideas sobre esta importante materia".

El documento de 3 de agosto de 1828, firmado por un grupo de siete favorecidos con mayorazgos, cuyos apellidos nos llegan hasta hoy, reaccionaba a la propuesta de nueva Constitución cuyo artículo 126 promulgaba la abolición invalidando las bases de la República e instalaba una argumentación sobre el derecho de propiedad completamente vigente en nuestra sociedad, uno que asocia este principio al de la seguridad, un derecho preferente al de la igualdad²:

"Por que el Congreso Constituyente es incompetente para dictar leyes de este jénero.

"Por que la lei de mayorazgos no es lei fundamental ni propia de una Constitución" "El tiempo anunciará, que cuanto mas se respete el principio de la propiedad, tanto mas se afirma en el espíritu del pueblo. Los pequeños atentados con este principio preparan los mayores. Ha sido necesario que pase mucho tiempo para llevarle al punto en que le vemos en las sociedades civilizadas, pero una fatal experiencia nos ha hecho conocer con cuanta facilidad puede ser alterado, y mirar con horror las desastrosas consecuencias de tales alteraciones".

"Porque cuando la seguridad y la igualdad están en oposición, no se debe dudar un momento, la igualdad es la que debe ceder, porque la primera es el fundamento de la vida: subsistencia, abundancia; felicidad, todo depende de ella; pero la igualdad no produce mas que una porción de bienestar".

La Constitución de 1833 avanzó en la propuesta de propiedades libres estableciendo un pago equivalente a la valorización de las tierras. Sólo en 1852 se declaró definitivamente abolida la institución del mayorazgo y una nueva ley, en 1857, confirmó su extinción y las propiedades pasaron a regirse por el nuevo Código Civil. La relación entre institucionalidad normativa de la propiedad de la tierra y economía es un binomio que tendrá en el Estado un ente mediador, regulador y expropiador de acuerdo con los proyectos de desarrollo que políticamente lograron imponerse y ganar en el Congreso durante el siglo XX. La tesis de Juvenal Hernández, Rector

2. Una respuesta a este libelo se puede leer en Memoria Chilena, firmado por los 11 restantes mayorazgos que, con iguales apellidos, estaban del lado de la Revolución, incluyendo a dos mujeres: Pedro Prado Jara Quemada, Martín de Larraín, José Toribio Larraín, José Miguel Bascuñan y Ovalle, Juan Agustín Alcalde, Francisco Ruiz Tagle, José Antonio Valdés, José Nicolás de la Cerda, Juan de Dios Correa, Agustina Rojas y Mercedes Rojas. 
de la Universidad de Chile entre 1933 y 1953, cuyo tema fue la expropiación por causa de utilidad pública, permite revisar las teorías respecto de la propiedad por un lado, y los mecanismos de indemnización por el otro. Nos pareció por sobre todo un aporte el concepto de "lo público" que allí se moviliza en particular en un nuevo contexto constitucional inaugurado por la Constitución de 1925 y nuestro incipiente Estado de protección de derechos sociales ${ }^{3}$.

Es importante dar nombre a los sujetos, hombres y mujeres, habitantes de dichas propiedades que con los estudios sociales de los años 1950 y 1960 pasaron a ser los campesinos o los proletarios del campo, los antiguos inquilinos que sustentaban la producción de los latifundios o que, simplemente, resguardaban los linderos de la propiedad bajo un régimen abusivo sin relaciones salariales sino que propiamente señoriales, como lo muestra la selección de tres cartas de puño y letra compiladas por el historiador Brian Loveman en El campesino chileno le escribe a su excelencia bajo el sello de los estudios de Icira ${ }^{4}$. Las cartas de los campesinos al presidente permiten visibilizar las complejidades y riqueza de los procesos sociales, pues las prácticas políticas de Antiguo Régimen permiten movilizar a los sujetos populares en el marco del derecho de petición, el amparo del poderoso hacia el desvalido y la denuncia de las injusticias como las bases de la revuelta social. Son llamados que no sabemos si conmovieron los oídos. Rita Tirón interpela al Presidente Eduardo Frei, "A ti presidente de los campesinos..." y antes que ella Pedro Coronado escribe al Presidente Juan Antonio Ríos pidiendo amparo al "primer mandatario del país", y Dominga Puebla acude al Presidente González Videla para denunciar las "maldades" de los dueños de las tierras".

El tercer conjunto de textos nos permite ver de qué forma la Universidad de Chile -como institución- aportó a los procesos con estudios e investigaciones necesarias, incorporando las nuevas ciencias sociales a su estructura, en la forma de institutos que dieron paso a las Facultades de Economía y Ciencias Sociales, las que justamente por su sesgo transformador estructural fueron eliminadas con la intervención militar. Los textos también son representativos del laborioso y contundente trabajo intelectual de los nuevos cientistas sociales latinoamericanos, animados por configurar un modelo de desarrollo propio. El primero de ellos, de

3. Juvenal Hernández. De la espropiación por la causa de utilidad pública. Imprenta Comercial. Santiago. 1923. Colección Tesis. Biblioteca Central de la Facultad de Derecho. Universidad de Chile.

4. Al igual que el clásico y no superado estudio de Mario Góngora sobre el Origen de los inquilinos del Valle Central.

5. Agradezco a Tomás Cornejo, Coordinador del Área de Investigación Patrimonial del Archivo Central Andrés Bello, la sugerencia de incluir estos valiosos documentos encontrados por el destacado historiador Brian Loveman en una publicación de poca circulación hoy. 
Eduardo Hamuy Berr (-1989), por ejemplo, ayudó a fundar en 1957 en la entonces Facultad de Filosofía y Educación, el Instituto de Sociología, para crear en 1964 el Centro de Estudios Socioeconómicos (CESO) en la Facultad de Economía. En torno a la Reforma Agraria, editado por el Instituto de Sociología de la Universidad de Chile en 1958, fue leído originalmente como ponencia en el primer congreso sobre Reforma Agraria en 1958 de la Facultad de Economía de la Universidad Central de Venezuela. El anuncio que hicieran los propietarios de 1826 parece una profecía, pues de acuerdo con Hamuy, efectivamente se transformaron en latifundistas empresarios y verían todo intento de cambio en el agro como una catástrofe:

"Por otra parte, la resistencia al cambio del sector propietario es tan fuerte que -hasta el momento- los ejemplos que poseemos (México, Bolivia, Cuba) revelan que la Reforma Agraria se realiza cuando se produce la crisis política de toda la sociedad.

¿Por qué esto es así? Probablemente porque las fuerzas sociales (grandes propietarios) cuyo poder está basado justamente en la agricultura, no han encontrado -en términos de poder social- la adecuada compensación que les permita renunciar a los privilegios que obtienen de una agricultura atrasada. La "modernización" de la agricultura -sobre la base de sus propios predios y de ellos mismos como empresarios - no es para la clase de los latifundistas una buena solución, porque perderían su posición estratégica en la estructura de poder de toda la sociedad. Planteadas así las cosas, la salida de esta situación no puede ser sino catastrófica".

Otro punto para rumiar de su propuesta es que a esta élite le falta "ascésis capitalista", le falta ahorro y capitalización y su ideología está cimentada en elementos tradicionales. No tienen contrapeso pues la "burguesía" existente es débil, vacilante e "inclinada" al compromiso político y económico: "Un factor que acentúa esta vacilación es el apoyo de las grandes potencias a la clase tradicional; no hay muchas evidencias que EE.UU. por ejemplo, mire con especial simpatía los esfuerzos nacionales por el desarrollo económico y, en cambio, hay muchas evidencias de que prefiere en el poder a la clase tradicional, sea con un dictador o sea con un presidente formalmente electo" (p.79). Muy interesante es, en este escenario, el papel que le otorga a la educación como catalizador de estos valores "ascéticos", aunque difícilmente podría tener la fuerza de la religión (protestante, pues sigue los análisis de Weber sobre el origen del capitalismo): "Una educación al servicio de la sociedad trataría de internalizar en los sujetos la idea que el consumo conspicuo es anti-social y que debe ser motivo de censura más bien que fuente 
de prestigio social." (p.80). El otro contrapeso sería la "mística socialista" en un sentido amplio.

Continuamos con el estudio del joven ingeniero comercial David Alaluf, publicado por el Instituto de Economía de la Universidad de Chile en 1960, relativo a la subdivisión de la propiedad agrícola en una región de la zona central de Chile. Cuenta con la presentación de Joseph Grunwald (1920- ) invitado a participar en la reestructuración del Instituto de Economía en el marco del convenio ChileCalifornia entre 1954 y 1961', cofundador de la Escolatina, primer programa de graduados en Economía en Latinoamérica, así como la Encuesta de Empleo del Gran Santiago, una iniciativa pionera en la región que ha estado vigente por más de 50 años. De David Alaluf sabemos que era parte del equipo de Icira, de los más jóvenes entre otros como Jorge Echenique, Sergio Gómez, Emilio Klein, Eugenio Maffei, Andrés Pascal, Alejando Saavedra, Alexander Schejtman y Hugo Zemelman, y que se formó junto a otros intelectuales latinoamericanos de gran renombre que laboraban en Icira, como el maestro Paulo Freire, de Brasil, y Antonio García, de Colombia.

Alaluf es representativo de los nuevos estudios económicos en el cruce de las ciencias del derecho y de la ingeniería tan fundamentales para los modelos de planificación y desarrollo de los nuevos estados de la segunda mitad del siglo XX, también ya en el contexto de la Guerra Fría y de la ola revolucionaria con influencias marxistas. El estudio de Alaluf vuelve a ratificar lo que se anunciaba a los debates de la Independencia: el 45\% de los predios eran adquiridos por herencia, situación que no sólo reforzaba el latifundio como vínculo tradicional, sino que también provocaba otro fenómeno negativo para la activación de la producción agrícola, que era el parvifundio. Los planos y estadísticas presentadas por el estudio permiten visualizar dicha realidad en el lenguaje moderno de las ciencias sociales.

El último texto es de autoría de Antonio García Nossa (1912-1982), investigador, historiador, académico, sociólogo, economista y político colombiano fundador de las investigaciones sobre los problemas agrarios y las características estructurales de la economía en muchos países de Latinoamérica. En 1945 fundó el Instituto de Ciencias Económicas de la Universidad Nacional, colaboró con la fundación del Instituto

6. Austríaco, debió huir de su país muy joven por la persecución nazi a los Estados Unidos, se nacionalizó y se enroló para pelear en la Segunda Guerra Mundial. Al volver se doctoró en Economía en la Universidad de Columbia. Después de su fructífera estancia en Chile, el profesor Grunwald sostuvo una exitosa carrera académica en la Universidad de Yale (1961), Brookings (1963), y la Universidad de California en San Diego (UCSD), llegando a ser el presidente fundador del Institute for the Americas (1984). 
Nacional Indigenista en dicho país y fue profesor del Instituto de Capacitación en Investigación en Reforma Agraria (Icira), proyecto conjunto del Gobierno de Chile y de las Naciones Unidas. Su análisis sobre las cooperativas del campo chileno, tema del que trata el texto seleccionado, era que estas instituciones estaban comprometidas esencialmente con el statu quo, esto es, una modernización periférica que no afectara al latifundio ni al minifundio, que carecían de autonomía para desempeñar un lugar en proyectos estratégicos, que tampoco servían a colonos, minufundistas y peones para su desarrollo a nivel comunal o integrarlos al sistema nacional de mercado o representativos del Estado. En definitiva, estas cooperativas no eran el sistema que permitiría "instrumentar un cambio estructural como la Reforma Agraria". En conclusión "es evidente que semejante formulación supone y exige un cambio muy profundo en la concepción misma del cooperativismo, ya que de lo que se trata no es de reproducir patrones organizativos o ideológicos norteamericanos o europeos, sino de diseñar una estructura que responda satisfactoriamente a las exigencias del desarrollo económico y sociales de la nación chilena, a las necesidades de organización multiactiva del campesinado (reeducación social, formación empresaria, canalización de servicios asistenciales del Estado), a la democratización del poder rural y al reto de los cambios estructurales” (p.12).

Su análisis del Estado chileno nos parece notablemente conocido al día de hoy: gran cantidad de recursos institucionales destinados a la fiscalización y al financiamiento del cooperativismo, pero sin modificar su carácter, y carencia de coherencia, articulación y capacidad para actuar unificadamente al no existir planificación de la acción del Estado, "ni siquiera un método de coordinación para actuar de las políticas desarrolladas en una misma línea operacional. Ni los organismos que operan en el campo del financiamiento -como el Banco del Estado y CORFO- ni aquellos que desenvuelven una nueva orientación en la política asistencial del Estado -como INDAP y CORA- disponen de los más elementales mecanismos de relación y o de coordinación de líneas operacionales. La acción del Estado (tan fundamental en América Latina pero mucho más en un país, como Chile, en el que los sectores estatales controlan el $70 \%$ de los recursos de inversión nacional), operan en un sentido vertical y sectorializado, por medio de órganos estancos y sin relaciones institucionales entre sí, retrasando las posibilidades de que esa acción pública pueda originar un equilibrado proceso de cambios" (p.13).

Alaluf y García se encuentran separados por una década de cambios y grandes diferencias intelectuales y políticas que derivaron en verdaderos cismas sociales y también universitarios, pues la Reforma Universitaria iniciada en 1968 tuvo en la Facultad de Ciencias Económicas hondas repercusiones, y de allí surgió la idea de 
crear una Facultad de Ciencias Sociales que sería conocida como el proyecto de la sede norte. En 1970 los que apoyaban tal idea se tomaron la Escuela en la calle República y colgaron un lienzo que decía “aquí está la sede de la nueva Facultad de Ciencias Sociales". Los que no apoyaban tal idea crearon la sede occidente, donde se quedó la formación más tradicional en materias económicas. La sede norte giró hacia el marxismo y la teoría de la dependencia, acogió el desarrollo de la sociología y la economía socialista. La división se produjo formalmente en 1972, entre tanto ambas sedes funcionaron con cátedras paralelas. La sede occidente se fue a la calle Pedro de Valdivia e incluso se separó la biblioteca. Luego del golpe de Estado la sede norte fue clausurada, era Decano José Elías.

\author{
Alejandra Araya Espinoza \\ Historiadora \\ Directora Archivo Central Andrés Bello \\ Vicerrectoría de Extensión y Comunicaciones \\ Universidad de Chile
}

